\title{
Cuidados paliativos e teoria humanística na enfermagem
}

\section{Palliative care and humanistic theory in nursing}

\author{
Tânia Cristina Schäfer ${ }^{1}$ Vasques Valéria Lerch Lunardi ${ }^{2} \bullet$ Priscila Arruda da Silva ${ }^{3}$ \\ Karen Knopp de Carvalho ${ }^{4}$ Simone Algeri ${ }^{5}$
}

\begin{abstract}
RESUMO
Refletir acerca da importância da utilização dos cuidados paliativos (CP) no cotidiano de trabalho dos trabalhadores de enfermagem à luz da abordagem teórica humanística de Paterson e Zderad. CP tem por finalidade proporcionar ao paciente fora da possibilidade de cura e com risco de vida e sua família melhor qualidade de vida. Quando o trabalhador de enfermagem, no seu processo de cuidar, aplica o referencial da Teoria Humanística em combinação com a terapêutica do CP, é possível identificar cada ser como existência única e singular, propiciando entender seu significado e compreendê-lo no decorrer da progressão de sua doença. Também, possibilita auxiliar tais trabalhadores a assistirem o paciente terminal no seu processo de finitude. Portanto, o desafio maior é mobilizar, nesses profissionais, a necessidade de reflexão sobre sua prática, buscando o comprometimento com a temática da terminalidade e dos $\mathrm{CP}$, tanto no seu processo permanente de formação, quanto da gestão institucional com a capacitação dos mesmos.
\end{abstract}

Palavras-chave: Cuidados Paliativos; Enfermagem; Ética; Humanização da Assistência.

\begin{abstract}
Aimed to reflect on the importance of the use of palliative care (PC) in the daily work of nursing staff in the light of humanistic theoretical approach of Paterson and Zderad. CP aims to provide the patient off the possibility of healing and life-threatening and your family a better quality of life. When the nursing workers in their care process, apply the Humanistic Theory in combination with therapeutic CP, you can identify each person as a unique and singular existence, allowing to understand their meaning and understand it during the progression of their disease. Enables also assist such workers to assist terminally ill patients in their process of finitude. Therefore, the biggest challenge is to mobilize, these professionals need to reflect on their practice, seeking compromise with the theme of the terminally ill and CP, both in their continuous training process, as the institutional management with the training of the same.
\end{abstract}

keywords: Palliative Care; Nursing; Ethics; Humanization of Assistance.

I Enfermeira, Prefeitura Municipal do Arroio dos Ratos, Brasil. Doutora em enfermagem pela Universidade Federal do Rio Grande. Mestre em Enfermagem pela Enfermagem Federal do Rio Grande.Tem experiência na área de Enfermagem do adulto, com ênfase em Enfermagem e Saúde, atuando principalmente nos seguintes temas: enfermagem, cuidados paliativos, ética, equipe de saúde e paciente/família no processo de morrer, em ambiente hospitalar e domiciliar. Atualmente é Enfermeira da Prefeitura Municipal do Arroio dos Ratos. Recebeu $3^{\circ}$ Lugar apresentação oral-III Congresso de Cuidados Paliativos do Mercosul, III Jornada da Liga Interdisciplinar de Cuidados Paliativos da UFPEL, I Simpósio de Cuidados Paliativos e medicina de família, Universidade Federal de Pelotas-UFPEL.

2 Professora aposentada, Universidade Federal do Rio Grande, Rio Grande do Sul, Brasil. Doutora em Enfermagem pela Universidade Federal de Santa Catarina. Mestre em Educação pela Universidade Federal do Rio Grande do Sul. Estágio de pós-doutorado na Universidade de Toronto (2002). Foi membro da Comissão de Avaliação da Área da Enfermagem na CAPES, Coordenadora do Comitê de Assessoramento da Enfermagem no CNPq, Membro do Comitê Assessor de Ciências da Saúde da FAPERGS (2006-2009) Coordenadora Adjunto do Comitê Assessor de Ciências da Saúde na FAPERGS, Bolsista de Produtividade do CNPq. Possui 260 artigos publicados em revistas indexadas.Atualmente, é Professora Associada da FURG aposentada, Professora Permanente voluntária dos Programas de PósGraduação em Enfermagem Tem experiência na área de Enfermagem, com ênfase em Ética na Enfermagem, Ética na Saúde e Educação em Enfermagem, atuando principalmente nos seguintes temas: enfermagem, trabalho da enfermagem, poder, ética e autonomia.

3 Universidade Federal do Rio Grande, Rio Grande do Sul, Brasil. Doutora em Enfermagem, Universidade Federal do Rio Grande, Rio Grande, Brasil. Mestre em Enfermagem pela Universidade Federal do Rio Grande. Estágio Pós-doutoral com financiamento pela Conselho Nacional de desenvolvimento científico e tecnológico (CNPq - Brasil) no período de 2015 a 20I7. Estágio Pós-doutoral pela Universidade Federal do Rio Grande, com bolsa financiada pela coordenação de aperfeiçoamento de Pessoal de Ensino Superior - CAPES (Brasil). Tem 52 artigos publicados em revistas indexadas. Atualmente integra os seguintes projetos de pesquisa: Perfil de Saúde dos Estudantes de Ensino Médio da Rede Pública e Particular de Rio Grande, RS, Brasil; EPI Rural Rio Grande: coorte de idosos da área rural de Rio Grande, Rio Grande do Sul; Saúde da população rural de Rio Grande, RS: um estudo sobre crianças e suas mães, mulheres em idade fértil e idosos. Projetos de extensão:Atendimento e Prevenção à Criança Vítima de Violência, $8^{\mathrm{a}}, 9^{\mathrm{a}}$ e $10^{\mathrm{a}}$ edição.

4 Professora titular, Universidade Federal de Pelotas, Brasil. Doutora em Enfermagem pela Universidade Federal de Rio Grande. Mestre em Enfermagem pela Universidade Federal de Pelotas. Atualmente é professora auxiliar pela Universidade Católica de Pelotas e técnica administrativa na Universidade Federal de Pelotas. Tem experiência na área da Enfermagem, com ênfase em Enfermagem em cuidados paliativos.

5 Professora Associada nível II, Universidade Federal do Rio Grande do Sul, Porto Alegre, Brasil. Doutora em Educação, Pontifícia Universidade Católica do Rio Grande do Sul. Mestre em Enfermagem pela Universidade Federal do Rio Grande do Sul. Especialista em Saúde Mental e Psiquiatria pela Universidade Federal do Rio Grande do Sul.Atualmente é professor associado nível II da Universidade Federal do Rio Grande do Sul.Tem experiência na área assistencial em Enfermagem Pediátrica e Enfermagem Psiquiátrica. Docente com regime de Dedicação Exclusiva da disciplina de Cuidado em Enfermagem ao recém-nascido, criança e adolescente. Pesquisadora na área de violência contra criança, com ênfase em violência intrafamiliar. Coordenadora do Projeto de Extensão Atendimento e Prevenção À Crianças Vítimas de Violência $10^{a}$ edição, Membro Efetivo do Programa de Proteção à Criança do Hospital de Clínicas de Porto Alegre. Membro da Comissão de Pesquisa da Escola de Enfermagem da Universidade Federal do Rio Grande do Sul. 


\section{INTRODUÇÃO}

O cuidado do paciente em processo de morte, ainda hoje, constitui-se em tema difícil e complexo de ser tratado, em especial para os trabalhadores de enfermagem, pela sua maior proximidade no cuidado prestado a esses pacientes. Salienta-se que ocorre um maior sofrimento para esses profissionais, pois, apesar de seus esforços refletidos em ações práticas, há perdas humanas' e uma maior exigência emocional por parte dos profissionais que trabalham e vivenciam a morte diariamente, muitas vezes, não sabendo como lidar com pacientes fora da possibilidade de cura e com risco de vida ${ }^{2}$.

Paralelo ao aumento da expectativa de vida, e consequentemente, ocorre o aparecimento, muitas vezes, de doenças fora da perspectiva de cura e com risco de vida. Dados estatísticos remetem que, em 2018,4I milhões, ou $72 \%$, das mortes ocorridas no mundo, foram decorrentes de doenças não transmissíveis, destacando-se as doenças cardiovasculares e pulmonares crônicas, diabetes e câncer $^{3}$. Apesar dos avanços tecnológicos na área da saúde, principalmente na oncologia, estima-se que, para 2020, I5 milhões de novos casos de câncer serão diagnosticados ${ }^{3}$.

Diante disso, os profissionais da saúde, em especial os de enfermagem, necessitam vivenciar um processo de sensibilização e capacitação quanto ao cuidado de pacientes fora da possibilidade de cura e com risco de vida, além de melhor proteger o seu estado psíquico e dos seres humanos assistidos, bem como estar prontos e motivados para orientar os familiares no enfrentamento desse momento sofrido, tanto físico, como emocional e muitas vezes financeiro ${ }^{4-5}$.

Dessa maneira, o profissional que convive com esses pacientes, diariamente, pode mobilizar sentimentos de impotência ou mesmo de fracasso em sua profissão, pois a perspectiva de cura da doença como uma meta específica a ser alcançada no cuidado que lhes é prestado não mais existe. Essa situação vivida costuma, lhes acarretar medo, angústia e sentimentos de finitude, podendo causar seu afastamento do paciente e de seu familiar cuidador, possivelmente por não saber o que e como falar e o que fazer nesse momento ${ }^{6}$.

Destarte, mesmo sem os trabalhadores perceberem, essa vivência pode acarretar situações consideradas desumanizantes no cuidado ao paciente, visto que o sofrimento do outro pode refletir-se como um espelho na vida desses profissionais, trazendo à luz dimensões da existência humana, que nem sempre querem lembrar, como a fragilidade, a vulnerabilidade e a mortalidade do ser humano ${ }^{7}$.

Para tanto, torna-se imprescindível que esses trabaIhadores estejam suficientemente preparados para atenderem a essa parcela da população. Nota-se, ainda, que dentre os profissionais da saúde que atuam junto a esses pacientes, os trabalhadores de enfermagem são os que mais ficam presentes no seu cuidado. Assim, deve haver um olhar para o cuidado solidário, que alia competências técnico-científicas e humanas, em meio à dor e ao sofrimento do outro. Simultaneamente, os trabalhadores de enfermagem podem e devem deixar-se tocar em sua sensibilidade, humanizando-se nesse processo ${ }^{7}$.

A filosofia dos CP aproxima-se da filosofia dos cuidados humanizados, proporcionando dignidade aos pacientes e seus familiares. Segundo a Organização Mundial da Saúde (OMS), em uma definição revisada, CP constituem uma abordagem que promove a qualidade de vida de pacientes que enfrentam doenças que ameacem a vida, e seus familiares, por meio da prevenção e do alívio do sofrimento. Requerem identificação precoce, avaliação e tratamento da dor e de outros problemas de natureza física, psicossocial e espiritual ${ }^{3}$.

A partir dessa definição, a OMS engloba princípios considerados fundamentais colaborando para nortear o trabalho dos profissionais da saúde, dentre eles: proporcionar alívio da dor e de outros sintomas angustiantes e melhorar a qualidade de vida; afirmar a vida e considerar a morte como um processo normal, sem apressá-la ou adiá-la; oferecer um sistema de apoio para ajudar os pacientes a viver tão ativamente quanto possível até a morte; utilizar uma abordagem de equipe para atender às necessidades dos pacientes e suas famílias, incluindo aconselhamento de luto, se indicado ${ }^{3,8}$.

Dessa forma, a teoria Humanística proposta por $\mathrm{Pa}-$ terson e Zderad ${ }^{9}$, vem ao encontro da filosofia dos CP, no sentido de instrumentalizar, construir e levar a reflexão do profissional nesse seu fazer diário, investindo no bem estar e em uma vida mais plena, visualizando a condição de vida e morte de cada paciente, valorizando seu potencial e condição humana. Nesse processo de cuidar no viver e no morrer, é preciso que sejam vistas todas as respostas possíveis do homem em sua situação, tanto de interesse e alegria, como de tristezas e sofrimentos e saber como lidar com essas situações?.

Desse modo, pode-se salientar que, para compreender e aderir aos princípios em CP mostra-se necessário rever o ambiente interno de cada trabalhador, sua percepção de cuidado, de si e do outro, de modo que não realize ações automáticas, mas antes possa sensibilizar-se com a dor do outro, proporcionando-lhe um atendimento qualificado e digno. Nesse sentido, o estudo teve por objetivo refletir acerca da importância da utilização dos CP no cotidiano laboral dos trabalhadores de enfermagem, à luz da abordagem teórica humanística de Paterson e Zderad.

\section{MÉTODO}

Trata-se de um estudo reflexivo, embasada em autores que abordam a temática acerca dos cuidados paliativos e sua relação com os elementos atribuídos ao 
conceito e abordagem, na área da enfermagem, a fim de delinear suas conexões com a Teoria Humanística de Paterson e Zderad.

Foram consultados artigos científicos pesquisados nas bases de dados LILACS (Literatura Latino-Americana e do Caribe em Ciências da Saúde) e na biblioteca virtual da SciElo (Scientific Electronic Library online) tendo como subsídio autores que abordam a temática. Após a leitura procedeu-se a análise descritiva e de conteúdo, o que contribuiu para a reflexão sobre a temática, a partir de três categorias: a) cuidados paliativos como uma filosofia humanitária; b) abordagem teórica humanística de Paterson e Zderad; c) cuidados paliativos à luz da teoria humanística.

\section{RESULTADOS}

\section{Cuidados Paliativos Como Uma Filosofia Huma- nitária}

Os primeiros movimentos para a implantação de CP, no Brasil, ocorreram no início da década de 1960, quando predominava uma modalidade hospitalocêntrica, voltada para a cura das doenças, com o cuidado multiprofissional, porém com diferentes profissionais, que não se comunicavam entre si a respeito das condutas adotadas em relação aos pacientes. Muitos pacientes hospitalizados eram oncológicos que vivenciavam dores intensas, morrendo solitários, em enfermarias com leitos separados por biombos e sem a presença de um ente querido nessa hora sofrida ${ }^{10}$.

O conceito de cuidados paliativos foi introduzido por Cecily Saunders que, ao fundar em Londres o St. Chirstopher Hospice, descreveu a filosofia do cuidar dos indivíduos terminais com diagnóstico de doença incurável, tendo como destaque o conforto dos pacientes e seus familiares" ${ }^{\prime}$. Com base em quatro elementos, considerado por ela como fundamentais (dor física, dor psicológica, dor social e dor espiritual, repudiava práticas que até então eram empregadas no tratamento a pacientes sem perspectiva de cura, muitas vezes caros e invasivos. Para Cecily, o cuidado deve ser pautado no conforto do paciente e familiares, na tentativa de obter qualidade de vida".

O objetivo dos cuidados paliativos é atingir a melhor qualidade de vida possível para os pacientes e suas famílias, tendo como filosofia: (I) afirmar a vida e encarar o morrer como um processo normal; (2) não apressar nem adiar a morte; (3) procurar aliviar a dor e outros sintomas angustiantes; (4) integrar os aspectos psicológicos e espirituais nos cuidados do paciente; (5) oferecer um sistema de apoio para ajudar os pacientes a viver ativamente tanto quanto possível até a morte; (6) disponibilizar um sistema de apoio para ajudar a família a lidar com a doença do paciente e com o seu próprio luto'2.

Apesar da prática dos CP existir já há algumas décadas e ter amplo desenvolvimento em muitos países, como no Reino Unido que, desde 1987, considera a medicina paliativa como uma especialidade médica ${ }^{13}$, a adesão dos profissionais da saúde, aos $\mathrm{CP}$ ainda é reduzida no Brasil. O difícil acesso aos serviços de assistência, faIhas nas diretrizes das políticas de saúde, deficiência na formação dos profissionais e o próprio desconhecimento dessa temática pela população em geral ${ }^{14}$ continuam sendo alguns dos entraves a serem transpostos para a implantação e consolidação desse cuidado.

Dessa forma, salienta-se que, mesmo de forma lenta, a filosofia dos CP vem se desenvolvendo. No entanto, é fundamental ampliar a discussão e a formação em CP, aprimorando os currículos dos cursos de graduação da área da saúde, em especial da área da enfermagem, com disciplinas que tratem essa temática com maior ênfase e profundidade, assim como no processo de educação permanente ${ }^{15}$. Faz-se necessário, também, investir na conscientização da própria população que pouco conhece e pouco discute a temática, acontecendo de a experiência ocorrer somente na prática, o que dificulta tanto a implantação desses serviços, como o trabalho das equipes de uma maneira geral.

\section{Abordagem Teórica Humanistica De Paterson E Zderad}

A Teoria Humanística surgiu na década de 1970, a partir das vivências das enfermeiras Josephine Paterson e Loretta Zderad no campo da docência e da assistência em Enfermagem, culminando na publicação de seu livro, no ano de 1976, com a descrição da construção e elaboração dessa teoria de enfermagem. Tal abordagem teórica apresenta a prática com um enfoque fenomenológico, humanista e existencial, a fim de auxiliar os trabalhadores de enfermagem a aprimorarem seus conhecimentos profissionais. Nessa direção, salienta-se que a enfermagem humanística é um despertar para as possibilidades de conformar nosso mundo da enfermagem aqui, agora e no futuro?.

Para essas teóricas, a enfermagem humanística se encontra em um ato humano em si, ou seja, o fenômeno da enfermagem tal como é vivido cotidianamente, ocorrendo, assim, o desenvolvimento inter-relacionado da teoria e da prática da enfermagem humanística, dependendo da bagagem de cada trabalhador, quanto a experiência, conceitualização e vivência particular no universo da enfermagem?. Propõe, também, uma enfermagem com base na experiência existencial, considerando a pessoa como um ser único e a soma de todos os seus compromissos e em um estado de vir a ser, com embasamento' na vivência dos enfermeiros que cuidam e do ser humano que recebe tal cuidado?.

Segundo as autoras, "Enfermagem Humanística" é mais que uma relação unilateral, mas antes uma relação transacional, cuja expressão demanda a conceituação embasada na consciência existencial que o trabalhador tem de si 
mesmo e do outro'. Portanto, consideram o cuidado em enfermagem como um encontro vivido e dialogado.

A percepção que se tem é que os enfermeiros precisam ter aptidão para perceber as necessidades e atitudes do ser cuidado, a fim de responder a elas, atentando para todo o contexto sociocultural em que praticam. Assim sendo, o encontro do enfermeiro com o paciente/ familiar é intencional um diálogo humano, quando um ser humano ajuda outro ser humano ${ }^{16}$.

Então, a utilização dessas palavras, "um ser humano ajuda outro ser humano", traduz-se em diálogo vivido a partir do cotidiano das ações de enfermagem, que, em geral, está tão envolvida com demandas imediatas do "fazer com" o paciente, que não se enfoca na atenção das correntes inter-humanas. Tal relação pode resultar em efeitos tanto humanizantes, mediante escuta e valorização dos desejos e sentimentos do outro, a fim de planejarem, enfermeiro, paciente, e, quando necessário, familiares, o cuidado adequado para a situação do paciente; quanto desumanizantes, quando o trabalhador não vai além de sua competência técnica e domínio biológico, na relação com o outro'.

\section{Cuidados Paliativos À Luz Da Teoria Humanística}

A enfermagem, para Paterson e Zderad ${ }^{9}$, é como uma resposta aos males da condição humana que ocorre em determinadas situações: quando um ser humano (paciente) necessita de um certo tipo de ajuda e o outro (trabaIhador de enfermagem) a proporciona. Para entender o significado da enfermagem como ato humano, é necessário considerá-la como um existente, ou coisa em si, um fenômeno que ocorre no mundo real das vivências humanas.

Ainda, a enfermagem é uma profissão que participa da vida dos indivíduos em várias etapas de sua existência (do nascer ao morrer) podendo ser uma resposta aos males da condição humana, através do processo de cuidado ${ }^{17}$. Então, os $\mathrm{CP}$, na enfermagem, podem visualizar o ser humano na sua individualidade, em seu processo de finitude, percebendo suas necessidades e agindo para que se respeite a dignidade nessa fase da vida ${ }^{18}$. Portanto, respeitar o momento vivido por esses pacientes, a singularidade de cada um, é uma forma de relacionamento autêntico que os trabalhadores de enfermagem humanística pretendem vivenciar ${ }^{18}$. Também, a busca por significados das experiências vivenciadas, a reflexão consciente e a valorização dessas experiências, podem ser fatores que desencadeiam o processo de desenvolvimento do potencial humano do trabalhador de enfermagem em busca de seu estado melhor ${ }^{19}$.

Para Paterson e Zderad ${ }^{20}$, os elementos do marco de referência estabelecido na Teoria Humanística de Enfermagem para promover a saúde incluem os seres humanos (trabalhadores de enfermagem e cliente) que se deparam em um encontro do ser e vir a ser, com um fim determinado, possibilitando nutrir o bem-estar e o estar melhor, em uma transação intersubjetiva do ser com e fazer com, acontecendo no tempo e espaço, delimitado e vivido pelo cliente e profissional de enfermagem em um mundo de homens e coisas. Portanto, é importante verificar que as principais dimensões da enfermagem humanística derivam da situação humana que o individuo está vivenciando, por exemplo, como em pacientes fora da perspectiva de cura e com risco de vida, que passam por uma situação difícil e sofrida.

Assim, na visão de Paterson e Zderad, o cuidado evidencia alguns conceitos básicos relacionados ao ambiente e à enfermagem, interligando a teoria à prática clínica, procurando descrever e compreender o vivido e o experenciado, tanto na forma como homem, como ser singular, como no seu âmbito coletivo, como se relaciona com o mundo e com o outro, valorizando-o, no intuito de conhecer suas potencialidades e limitações ${ }^{21}$. Para tanto, as palavras tornam-se instrumentos indispensáveis para a descrição fenomenológica, mesmo que limitadas pela habilidade do indivíduo em expressá-las; no entanto, se constituem no melhor meio para se expressar a condição humana?.

Dessa maneira, pode-se considerar que os trabalhadores de enfermagem, ao atuarem em CP, estimulam o potencial de coexperimentar e apoiar o processo vivido pelo paciente, a partir da filosofia da Teoria Humanística de enfermagem ${ }^{22}$. Assim, esse profissional e o paciente se humanizam ao fazerem escolhas responsáveis na situação intersubjetiva e transacional do cuidado, conduzindo a uma enfermagem humanística. Nesse contexto, ao assistir o paciente fora de possibilidade de cura e com risco de vida, os trabalhadores precisam respeitar o outro, simultaneamente, sendo solidários com o outro. É necessário ter sensibilidade e compaixão frente a sua dor, mantendo sua individualidade e dignidade, visto que só se consegue ser-com o enfermo visualizando-se sua unicidade, uma vez que cada indivíduo é único e singular ${ }^{23}$.

Salienta-se, ainda, que o cuidado humanizado envolve a presença verdadeira e legítima, o diálogo vivo e autêntico entre as pessoas. O estar com ou o estar ali, na realidade, é um tipo de relacionamento que implica a presença ativa do profissional de enfermagem, isto é, estar atento a uma abertura aqui e agora na situação de comunicar a disponibilidade, envolvendo o estar presente, que constitui uma chamada e uma resposta ${ }^{24}$. Esse relacionamento faz parte do conceito de ambiente que, na teoria humanística, é considerado de forma subjetiva, transcendente, além da concepção de espaço físico?.

A prática da enfermagem e sua fundamentação teórica estão inter-relacionadas, em que os trabalhadores de enfermagem são aqueles que desenvolvem sua prática como um ser humano que sente, valoriza, reflete e conceitua. Do 
cotidiano da enfermagem, surge o conjunto organizado do conhecimento clínico, formado na interação dialógica das experiências articuladas e compartilhadas pela equipe de enfermagem, investigadores que abstraem e conceituam? Da mesma forma, os CP precisam ser pensados e articulados no cotidiano de trabalho desses profissionais, no intuito de se programarem ações condizentes com cada paciente, no intuito de sempre beneficiá-lo, preservando sua autonomia e capacidade de tomar decisões ${ }^{25}$, buscando sanar suas necessidades e de seus familiares.

\section{CONCLUSÃO}

Através deste estudo teórico, apoiado na Teoria Humanística proposta por Paterson e Zderad, pode-se considerar que, a partir da filosofia dos $\mathrm{CP}$, o ser humano possa ser auxiliado em seu processo de morrer, tendo, como fio condutor do cuidado, a preservação da sua dignidade, uma vez que a assistência recebida ultrapassa um fazer técnico, envolvendo questões éticas, étnicas, culturais, humanas, sociais e espirituais. Dessa forma, o estudo mostrou, ainda, que o conhecimento dessa abordagem humanística pode auxiliar o trabalhador na discussão e reflexão e qualificação de seu cotidiano de trabalho, colaborando para o planejamento e desenvolvimento de ações que possibilitem não apenas sua instrumentalização, mas também a de familiares de pacientes que enfrentam doenças que ameacem a vida, para realizarem de forma mais efetiva o cuidado, em CP, a seus entes queridos, quando forem para o ambiente domiciliar.

A instrumentalização dos profissionais de saúde com conhecimentos específicos, acerca dos $\mathrm{CP}$, apontadas no estudo, constitui-se em elemento essencial que determina não só um cuidado de qualidade, mas principalmente mais humanizado.Assim, o desafio maior instigado nesse estudo teórico é mobilizar, nos profissionais da saúde, em especial nos trabalhadores de enfermagem, por essa maior proximidade com o paciente e seu familiar no decorrer de sua lida diária, a necessidade de reflexão sobre a sua prática, buscando o comprometimento com a temática da terminalidade e dos CP, tanto no seu processo de formação permanente, quanto da gestão institucional com a capacitação de seus trabalhadores, qualificando o cuidado prestado aos pacientes no final da vida, e aos seus familiares, auxiliando-os no entendimento da filosofia dos CP, juntamente com uma visão da abordagem Teórica Humanística. As ações desses trabalhadores precisam ser, também, planejadas com o intuito de proporcionar ao indivíduo e à família uma experiência menos dolorosa. 


\section{REFERÊNCIAS}

I. Pereira SM, Pires EF.As experiências de perdas e luto na contemporaneidade: um estudo bibliográfico. Rev Educação [internet] 2018 [cited $2019 \mathrm{fev} \mathrm{I2];} \mathrm{I3(I):200-17.} \mathrm{Availa-}$ ble from: http://revistas.ung.br/index.php/educacao/article/ view/2837

2. Nunes FN, Araújo KM, Silva LDC.As evidências sobre o impacto psicossocial de profissionais de enfermagem frente à morte. R. Interd [internet]. 2016 [cited 2019 mar 20]; 9(4): 165-72. Available from: https://revistainterdisciplinar.uninovafapi.edu.br/index.php/revinter/article/view/545

3. World Health Organization (WHO). Who Definition of Palliative Care. [acesso 28 mar 2018] Disponível: http:// www.who.int/cancer/palliative/definition/en

4. Ouchi JD, Freitas GG, Ribeiro CAG, Oliveira CC. O impacto da morte para o profissional de enfermagem. Rev Saúde em Foco. 2018; I0(I):404-II.

5. Barbosa AGC, Massaroni L, Lima EFA. Significados do processo do morrer e da morte para a equipe multiprofissional. Rev Pesq Cuidado é Fundamental [internet] 2016 [cited 2019 mar 20]; 8(2):45 I0-7. DOI: I0.9789/2।75-536।.20I6. v8i2.45 I0-45I7.

6. Rodriguez, MF. Despedida silenciada: equipe médica, família, paciente - cúmplices da conspiração do silêncio. Psic. Rev. São Paulo [internet]. 2014 [cited 2019 fev I2]; 23(2):26I72. Available from: https://revistas.pucsp.br/psicorevista/article/view/2277///6503

7. Pessini L. Humanização da dor e do sofrimento humano na área da saúde. In: Pessini L, Bertachini L (org). Humanização e Cuidados Paliativos. Edições Loyola: $4^{\mathrm{a}}$ edição 2009.344p.

8. Prado RT, Leite JL, Silva IR, Silva LJ, Castro RAB.The process of dying/death: intervening conditions to the nursing care management. Rev Bras Enferm [internet] 2018 [cited $2019 \mathrm{fev}$ 20];7I(4):2005-I3. DOI: http://dx.doi.org/I0.I590/00347167-2017-0173

9. Paterson JG, Zderad LT. Enfermeria humanistica. Mexico: Editorial Mimusa, 1979.

10. Gomes ALZ, Othero MB. Cuidados paliativos. Estudos avançados [internet] 2016 [cited $2019 \mathrm{fev}$ I2]; 30(88): I55- 166. Available from: http://www.scielo.br/pdf/ea/v30n88/01034014-ea-30-88-0I55.pdf

I I. Brasil. Ministério da Saúde. Instituto Nacional de Câncer. Cuidados paliativos oncológicos: controle da dor. Rio de Janeiro: INCA; 200 I.

12. International Association for Hospice \& Palliative Care. Promoting Hospice \& Palliative Care Worldwide. The IAHPC Manual of palliative care, 2 nd edition.

13.Victor GHGG. Cuidados paliativos no mundo. Revista Brasileira de Cancerologia [internet] 2016 [cited $2019 \mathrm{fev} \mathrm{I2];62(3):}$ 267-270. Available from: http://wwwl.inca.gov.br/rbc/n_62/ v03/pdf/I I-resenha-cuidados-paliativos-no-mundo.pdf

14. Frossard A. Os cuidados paliativos como política pública: notas introdutórias. Cad. EBAPE [internet] 2016 [cited
2019 fev I2]; I4(spe): 640-655. Available from: http://www. scielo.br/pdf/cebape/vI4nspe/ I679-395 I-cebape- I4-spe-00640.pdf

15. Cruz RAO, Arruda AJCG, Agra G, Costa MML, Nóbrega VKM. Reflexões acerca dos cuidados paliativos no contexto da formação em enfermagem. Rev enferm UFPE on line. 20 16; I0(8): 3 I0I-7. https://periodicos.ufpe.br/revistas/revistaenfermagem/article/viewFile/I | 38 I/I3 | 30

16. Andrade CG, Costa SFG, Lopes MEL. Cuidados paliativos: a comunicação como estratégia de cuidado para o paciente em fase terminal. Ciênc saúde colet [internet] 2013 [cited 2019 fev I2]; I8(9):2523-30. Available from:

http://www.scielo.br/pdf/csc/vl8n9/vl8n9a06.pdf

17. Siqueira J, Zilli F, Oliveira SG. Profissionais de saúde e o processo de morte e morrer dos pacientes: uma revisão integrativa. pers. Bioét [internet] 2018 [cited 2019 mar 12]; 22(2): 288-302. DOI: 10.5294/pebi.2018.22.2.7

18. Calsavara VJ, Scorsolini-Comin F, Corsi CAC. A comunicação de más notícias em saúde: aproximações com a abordagem centrada na pessoa. Revista da Abordagem Gestáltica [internet] 2019 [cited 2019 mar 20]; XXV (I): 92-102. DOI: I0.18065/RAG.2019v25.9

19. Brito FM, Costa ICP, Costa SFG, Andrade CG, Santos KFO, Francisco DP. Communication in death imminence: perceptions and strategy adopted for humanizing care in nursing. Esc Anna Nery 20I4;18(2):317-322. DOI: 10.5935//4I48145.20140046

20. Paterson JG, Zderad LT. Humanistic nursing. New York: National League for Nursing; 1988.

2I. França JRFS, Costa SFG, Lopes MEL, Nóbrega MML, França ISX. Importância da comunicação nos cuidados paliativos em oncologia pediátrica: enfoque na Teoria Humanística de Enfermagem. Rev latino am enferm. 20I 3; 2 I (3):780-6.

22. Silva NRF, Farias DC, Souza JR, Bezerra FMC, Ferreira LS, Carvalho PMG. Teorias de enfermagem aplicadas no cuidado a pacientes oncológicos: contribuições para a prática clínica do enfermeiro. Rev Uningá 2018; 55(2): 59-7I.

23. Burlá C, Py L. Palliative care: science and protection at the end of life. Cad. Saúde Pública [internet] 2014 [cited 2019 mar I0]; 30(6): I |39-I |4I. Available from: http://www.scielo.br/pdf/csp/v30n6/0 I02-3 I IX-csp-30-6- I I 39.pdf

24. Siqueira JE, Pessini L. Reflections on care for critical patients at the end of life. Rev. bioét. [internet] 2019 [cited 2019 mar 20]; 27 (I): 29-37. Available from: http://www.revistabioetica.cfm.org.br/index.php/revista_bioetica/article/ view/ I 543/2009

25. Sousa KC, Carpigiani B. Ditos, não ditos e entreditos: a comunicação em cuidados paliativos. Psicologia:Teoria e Prática [internet] 2010 [cited $2019 \mathrm{fev} \mathrm{I2];} \mathrm{I2(I):97-I08.} \mathrm{Available}$ from: http://pepsic.bvsalud.org/pdf/ptp/vI2nI/vI2nla09.pdf

Recebido: 2019-07-17

Aceito: 2019-08-23 